\title{
Metabolic changes and diabetes microvascular complications 5 years after bariatric surgery
}

\author{
S.Ravindra, A.Miras, A.Humphreys, A.Ahmed, K. Moorthy, S.Purkayastha, S.Hakky, J.Cousins, S.Allen, T.Tan and
}

H.Chahal.

\section{Imperial Weight Centre, Imperial College London, U.K}

\section{BACKGROUND}

Bariatric surgery has pronounced effects on the metabolic profile of patients with type 2 diabetes mellitus (T2DM).

The American Diabetes Association (ADA) and International Diabetes Federation (IDF) have provided standardised definitions of T2DM remission.

Long-term remission rates of T2DM following bariatric surgery according to these criteria and its effects on T2DM microvascular complications remain unknown.

\section{OBJECTIVES}

1. To assess the rates of T2DM patients achieving the ADA and IDF criteria for T2DM remission 5 years after bariatric surgery.

2. To assess the long-term effect of bariatric surgery on albuminuria and retinal appearances in patients with T2DM using direct measures.

\section{METHOD}

Clinical data and direct measurements of renal and retinal damage were collected prospectively and analysed retrospectively for 82 patients with T2DM who underwent bariatric surgery (Roux-en- $Y$ gastric bypass, $n=59$; gastric banding, $n=8$; vertical sleeve gastrectomy, $n=15$ ) in a UK centre of excellence and were followed up for 5 years.

RESULTS

TABLE 2. Pre-operative and 5-year post-operative data from the cohort of patients studied ( $n=82$ ).

\begin{tabular}{|c|c|c|c|}
\hline & Pre-operatively & $\begin{array}{l}5 \text { years post- } \\
\text { operatively }\end{array}$ & $P$ value \\
\hline Weight (Kg) & $\begin{array}{c}133.5[113.7-150.0] \\
(84.0-235.0)\end{array}$ & $\begin{array}{c}100.4[82.2-115.0] \\
(62.0-165.0)\end{array}$ & $<0.0001$ \\
\hline \% weight loss & $\mathrm{n} / \mathrm{a}$ & $\begin{array}{c}24.0 \pm 11.8 \\
(-3.0-50.7)\end{array}$ & $n / a$ \\
\hline HbA1c (\%) & $\begin{array}{c}7.7[6.7-9.3) \\
(5.2-12.6)\end{array}$ & $\begin{array}{c}6.3[5.6-7.2] \\
(5.0-9.6)\end{array}$ & $<0.0001$ \\
\hline $\begin{array}{l}\text { HbA1c } \\
\text { (mmol/mol) }\end{array}$ & $\begin{array}{c}61.0[50.0-78.0) \\
(33.0-114.0)\end{array}$ & $\begin{array}{c}45.0[38.0-55.0] \\
(31.0-81.0)\end{array}$ & $<0.0001$ \\
\hline $\begin{array}{l}\text { Glucose } \\
\text { (mmol/l) }\end{array}$ & $\begin{array}{c}7.1[5.3-9.4] \\
(3.0-19.4)\end{array}$ & $\begin{array}{c}6.0[5.0-8.0] \\
(3.1-16.0)\end{array}$ & 0.0014 \\
\hline $\begin{array}{l}\text { Total cholesterol } \\
(\mathrm{mmol} / \mathrm{l})\end{array}$ & $\begin{array}{c}4.7[4.0-5.4] \\
(2.1-8.8)\end{array}$ & $\begin{array}{c}4.4[3.8-5.1] \\
(2.0-9.1)\end{array}$ & 0.21 \\
\hline $\begin{array}{l}\text { HDL cholesterol } \\
(\mathrm{mmol} / \mathrm{l})\end{array}$ & $\begin{array}{c}1.1[0.9-1.3] \\
(0.5-2.1)\end{array}$ & $\begin{array}{c}1.4[1.2-1.6] \\
(0.7-2.5)\end{array}$ & $<0.0001$ \\
\hline $\begin{array}{l}\text { LDL cholesterol } \\
\text { (mmol/l) }\end{array}$ & $\begin{array}{c}2.6[1.9-3.6] \\
(0.4-5.2)\end{array}$ & $\begin{array}{c}2.3[1.8-3.0] \\
(1.0-6.2)\end{array}$ & 0.16 \\
\hline $\begin{array}{l}\text { Triglycerides } \\
\text { (mmol/l) }\end{array}$ & $\begin{array}{c}1.8[1.3-2.4] \\
(0.6-7.4)\end{array}$ & $\begin{array}{c}1.3[0.9-1.8] \\
(0.4-4.4)\end{array}$ & $<0.0001$ \\
\hline Systolic BP (mmHg) & $\begin{array}{c}142[130-150] \\
(103-195)\end{array}$ & $\begin{array}{c}128[120-139] \\
(104-196)\end{array}$ & $<0.0001$ \\
\hline Diastolic BP (mmHg) & $\begin{array}{c}84[80-90] \\
(63-114)\end{array}$ & $\begin{array}{c}76[70-80] \\
(57-110)\end{array}$ & $<0.0001$ \\
\hline $\begin{array}{l}\text { Glucose lowering } \\
\text { medication }\end{array}$ & $\begin{array}{l}2[1-2] \\
(0-4)\end{array}$ & $\begin{array}{l}1[0-1] \\
(0-3)\end{array}$ & $<0.0001$ \\
\hline $\begin{array}{l}\text { BP lowering } \\
\text { medication }\end{array}$ & $\begin{array}{c}1[0-2] \\
(0-5)\end{array}$ & $\begin{array}{l}1[0-2] \\
(0-5)\end{array}$ & 0.48 \\
\hline $\begin{array}{l}\text { Lipid lowering } \\
\text { medications }\end{array}$ & $\begin{array}{c}1[0-1] \\
(0-3)\end{array}$ & $\begin{array}{l}1[0-1] \\
(0-1)\end{array}$ & 0.86 \\
\hline Hypoglycaemia (\%) & $\mathrm{n} / \mathrm{a}$ & $32 \%$ & $\mathrm{n} / \mathrm{a}$ \\
\hline ACR (mg/mmol) & $\begin{array}{l}1.8[0.8-4.9] \\
(0.2-312.4)\end{array}$ & $\begin{array}{c}1.2[0.7-4.3] \\
(0.1-413.4)\end{array}$ & 0.48 \\
\hline $\begin{array}{l}\text { ACR in cohort with } \\
\text { albuminuria at } \\
\text { baseline, } n=62 \text {, } \\
\text { (mg/mmol) }\end{array}$ & $\begin{array}{l}7.1[4.6-29.5] \\
(2.9-312.4)\end{array}$ & $\begin{array}{l}3.6[0.6-18.1] \\
(0.3-413.4)\end{array}$ & 0.020 \\
\hline
\end{tabular}

TABLE 1. The American Diabetes Association (ADA) and International Diabetes Federation (IDF) criteria

\begin{tabular}{|c|c|}
\hline & $\begin{array}{l}\text { ADA criteria definitions } \\
\text { Complete remission of Type } 2 \text { Diabetes Mellitus } \\
\text { - HbA1c }<42 \mathrm{mmol} / \mathrm{mol}(6.0 \%) \\
\text { - fasting glucose }<5.6 \mathrm{mmol} / \mathrm{l}(100 \mathrm{mg} / \mathrm{dl}) \\
\text { at least } 1 \text { year's duration in the absence of active pharmacologic therapy or ongoing } \\
\text { ADA complures. }\end{array}$ \\
\hline & $\begin{array}{l}\text { Partial remission of Type } 2 \text { Diabetes Mellitus } \\
\text { - HbA1c }<48 \mathrm{mmol} / \mathrm{mol}(6.5 \%) \\
\text { - fasting glucose } 5.6-6.9 \mathrm{mmol} / \mathrm{l}(100-125 \mathrm{mg} / \mathrm{dl}) \\
\text { at least } 1 \text { year's duration in the absence of active pharmacologic therapy or ongoing } \\
\text { procedures }\end{array}$ \\
\hline & IDF criteria definitions \\
\hline & $\begin{array}{l}\text { Optimisation of the metabolic state } \\
\text { - HbA1c } \leq 42 \mathrm{mmol} / \mathrm{mol}(6.0 \%) \\
\text { - no hypoglycaemia } \\
\text { - } \quad \text { total cholesterol }<4.0 \mathrm{mmol} / \mathrm{l} \\
\text { - } \text { triglycerides }<2.2 \mathrm{mmol} / \mathrm{l} / \mathrm{l} \\
\text { - } \quad>15 \% \text { weight loss } \\
\text { with reduced medication from the pre-operated state or without other medications (where } \\
\text { medications are continued, reduced doses from pre-surgery with minimal side effects } \\
\text { would be expected) }\end{array}$ \\
\hline & $\begin{array}{l}\text { Substantial improvement of the metabolic state } \\
\text { - } \\
\text { - } \quad \text { Lowering of } \mathrm{HbA} 1 \mathrm{c} \text { by }>20 \% \\
\text { blood pressure }<135 / 85 \mathrm{mmHg} \\
\text { with reduced medication from the pre-operated state }\end{array}$ \\
\hline
\end{tabular}

FIGURE 1. Percentage of patients that met the ADA and IDF criteria $(n=82)$

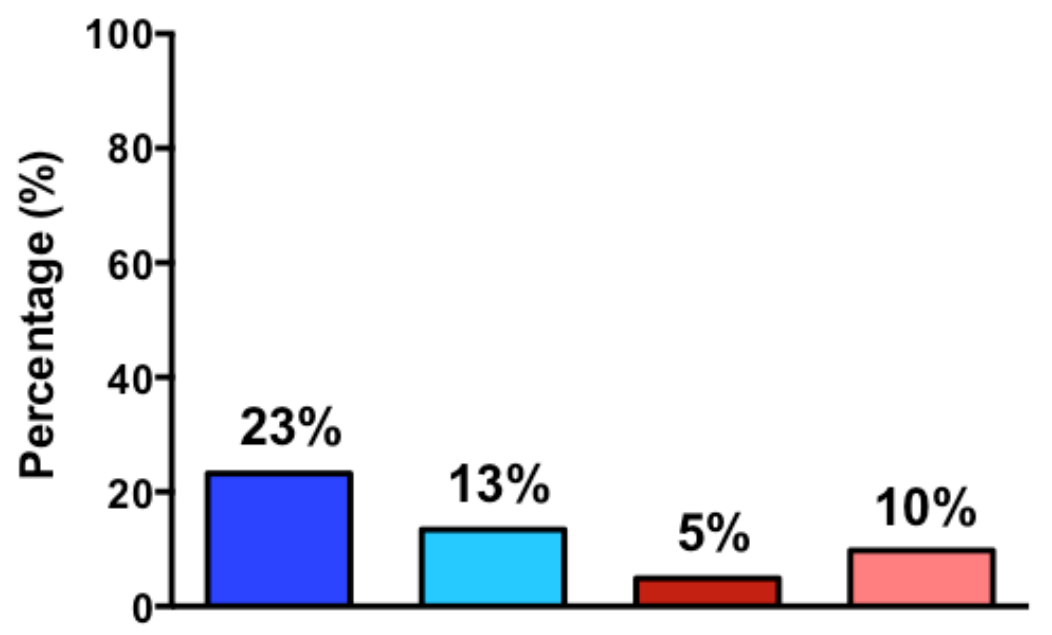

At 5 years, $23 \%$ and $13 \%$ patients achieved complete and partial remission of T2DM based on the ADA criteria respectively; $5 \%$ and $10 \%$ patients achieved optimisation and improvement of the metabolic state based on the IDF criteria respectively.

FIGURE 2. Retinal appearances pre- and post-operatively $(n=24)$

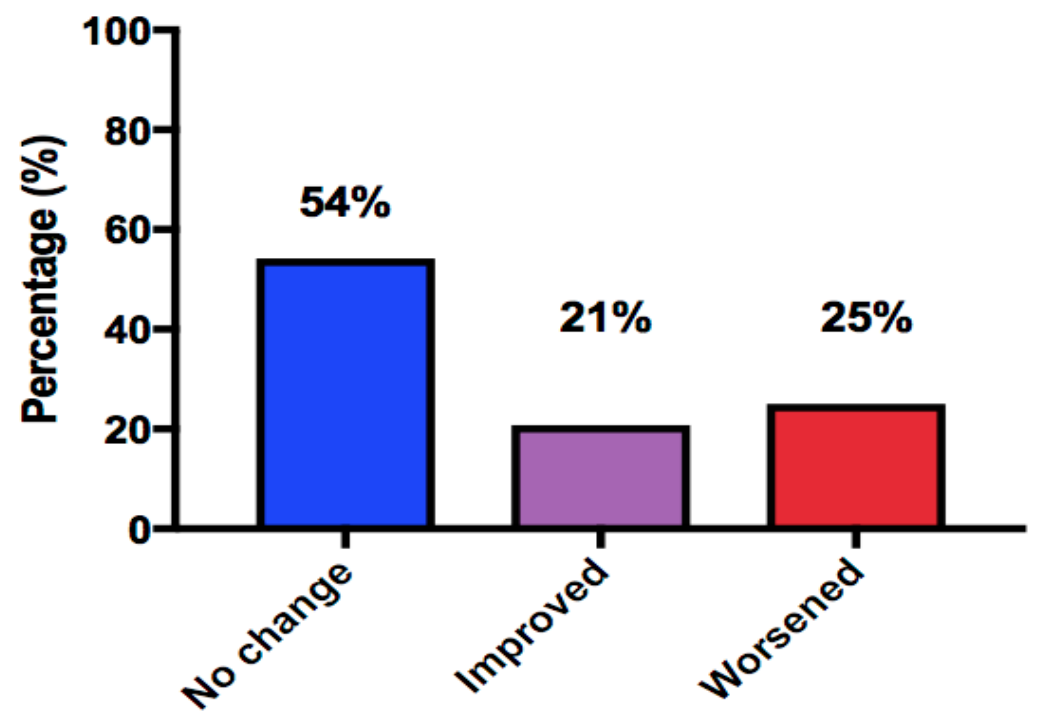

In the group of 24 patients with available retinal images, retinal appearances remained stable in 13 , worsened in 6 and regressed in 5 patients.

\section{CONCLUSIONS}

Bariatric surgery induced a significant sustained improvement of cardio-metabolic risk factors in patients with T2DM, however a relatively low number of patients achieved T2DM remission at 5 years. Whilst the findings on microvascular complications are encouraging, these results raise the hypothesis that concurrent intensive medical therapy may be required to achieve more stringent and optimal cardio-metabolic targets. Such an approach may further reduce the microvascular and macrovascular complications of T2DM. 